\title{
A highly efficient synthesis of an octasaccharide, the repeating unit of the cell-wall mannan of Trichophyton mentagrophytes and $T$. rubrum
}

\author{
Jun Ning, ${ }^{\mathrm{a}}$ Linsen Heng, ${ }^{\mathrm{b}}$ Fanzuo Konga,* \\ ${ }^{a}$ Research Center for Eco-Environmental Sciences, Chinese Academy of Sciences, PO Box 2871, Beijing 100085, PR China \\ ${ }^{\mathrm{b}}$ Daxian Normal College, Department of Chemistry, Sichuan, PR China
}

Received 25 March 2002; accepted 30 April 2002

\begin{abstract}
A highly concise and effective synthesis of the mannose octasaccharide repeating unit of the cell-wall mannan of Trichophyton mentagrophytes and T. rubrum was achieved via 6-O-glycosylation of a tetrasaccharide acceptor with a tetrasaccharide donor, followed by deprotection. The key tetrasaccharide (11) was constructed by selective 6-O-glycosylation of allyl 3,4-di- $O$-benzoyl- $\alpha$ D-mannopyranosyl-( $(\rightarrow 6)$-2,3,4-tri- $O$-benzoyl- $\alpha$-D-mannopyranoside with 6 - $O$-acetyl-2,3,4-tri- $O$-benzoyl- $\alpha$-D-mannopyranosyl trichloroacetimidate, then with 2,3,4,6-tetra- $O$-benzoyl- $\alpha$-D-mannopyranosyl trichloroacetimidate. The tetrasaccharide acceptor (13) was obtained by selective 6-O-deacetylation of 11, while the tetrasaccharide donor $\mathbf{1 2}$ was obtained by deallylation of $\mathbf{1 1}$, followed by trichloroacetimidation. (C) 2002 Elsevier Science Ltd. All rights reserved.
\end{abstract}

Keywords: Regio- and stereoselective synthesis; Mannopyranosyl oligosaccharides; Trichloroacetimidates

\section{Introduction}

The anthropophilic dermatophytes, Trichophyton mentagrophytes and T. rubrum, cause chronic, relatively uninflamed, skin infections of the feet (tinea pedis) and body (tinea corporis), often associated with infection of the nails (onychoycosis). About $90 \%$ of chronic dermatophyte infections are caused by T. mentagrophytes and T. rubrum, ${ }^{1}$ in part because these organisms can suppress inflammation and cell-mediated immunity. ${ }^{2}$ The cell-wall polysaccharides of these fungi are known to be the major immunologically active substances. There are mainly two kinds of polysaccharides present in the cell-wall, i.e., mannan and galactomannan. ${ }^{3}$ The mannan has a linear backbone consisting of $\alpha-(1 \rightarrow 6)$-linked mannose units, with $\alpha-(1 \rightarrow 2)$-linked mannose units as side chains, and its structure ${ }^{3}$ is shown as follows:

\footnotetext{
* Corresponding author. Tel.: + 86-10-62936613; fax: + 86-10-62923563

E-mail address: fzkong@mail.rcees.ac.cn (F. Kong).
}

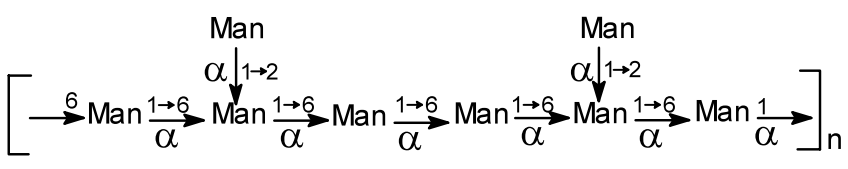

Blake et al. examined the ability of a cell-wall mannan isolated from T. rubrum to suppress cell-mediated immune reactions ${ }^{4}$ and found that the T. rubrum mannan inhibits the lymphoproliferative response of human mononuclear leukocytes to a variety of antigenic and mitogenic stimuli in vitro. Furthermore, Grando et al. demonstrated that the target cells for this effect of the mannan are monocytes and keratinocytes, by monitoring the binding and uptake of FITC-mannan using flow cytometry and fluorescence microscopy. ${ }^{5,6}$ To further elucidate the molecular structure responsible for the immunoinhibitory activity of the fungi mannan, it will be very useful to synthesize the repeating unit of the mannan. We report herewith a highly efficient and concise synthesis of the octasaccharide $\mathbf{1}$, the repeating unit of the cell-wall mannan of T. mentagrophytes and T. rubrum. 


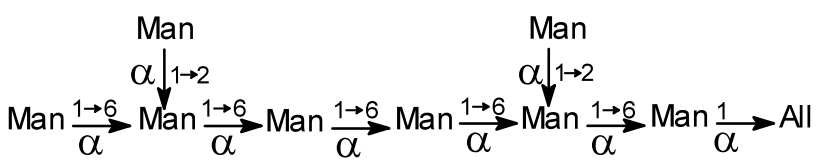

\section{Results and discussion}

First we synthesized the key synthon 6 (Scheme 1). Thus, tritylation of 1,2- $O$-( $R$-ethylidene)- $\beta$-D-mannopyranose $^{7}(2)$, followed by benzoylation in a one-pot manner, gave the 3,4-di- $O$-benzoyl-1,2- $O$ - $(R$-ethylidene)-6- $O$-trityl-D-mannopyranose (3). Acetolysis of 3 with $\mathrm{CH}_{2} \mathrm{Cl}_{2}-\mathrm{AcOH}-\mathrm{Ac}_{2} \mathrm{O}-\mathrm{H}_{2} \mathrm{SO}_{4}$ in a ratio of 1:1:0.6:0.18 afforded the 1,2,6-tri- $O$-acetyl-3,4-di- $O$ benzoyl- $\alpha$-D-mannopyranose (4). The triacetate 4 was selectively deacetylated at the anomeric position with benzylamine in THF in high yield to give the corresponding 2,6-di- $O$-acetyl-3,4-di- $O$-benzoyl-D-mannopyranose (5). Subsequent reaction of 5 with $\mathrm{CCl}_{3} \mathrm{CN}-$ $\mathrm{DBU}$ in dichloromethane afforded the glycosyl donor 6.

With the synthon 6 in hand, construction of the target compound was readily carried out. As shown in Scheme 2, the disaccharide 7 was prepared using $\mathbf{6}$ as the glycosyl donor and allyl 2,3,4-tri- $O$-benzoyl- $\alpha$-Dmannopyranoside as the acceptor. ${ }^{8}$ Selective removal of the acetyl groups of 7 in methanol solution containing $0.5 \% \mathrm{HCl}$ gave the diol glycosyl acceptor $8 .{ }^{8}$ Coupling of 8 with 6- $O$-acetyl-2,3,4-tri- $O$-benzoyl- $\alpha$-D-mannopyranosyl trichloroacetimidate ${ }^{8}$ as the glycosyl donor selectively gave the $(1 \rightarrow 6)$-linked trisaccharide 9 . Acetylation of 9 confirmed the 6-O-glycosylation as the ${ }^{1} \mathrm{H}$ NMR spectrum of acetylated trisaccharide $\mathbf{1 0}$ showed a newly emerged doublet of doublets at $\delta 5.67$ ppm for H-2. Condensation of 9 with 2,3,4,6-tetra- $O$ benzoyl- $\alpha$-D-mannopyranosyl trichloroacetimidate ${ }^{8}$ using TMSOTf as catalyst afforded the tetrasaccharide 11. The ${ }^{1} \mathrm{H}$ NMR spectrum of 11 showed one acetyl signal ( $\delta$ 1.96), allyl signals $(5.47-5.30)$, and four $\mathrm{H}-1$ signals $(5.33,5.20,5.05,4.91)$ characteristic of the

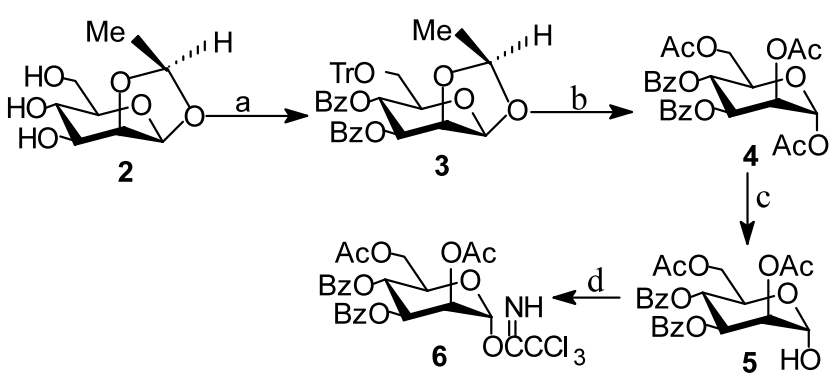

Scheme 1. Conditions and reagents: (a) i. trityl chloride (1.2 equiv), pyridine, $50{ }^{\circ} \mathrm{C}, 32 \mathrm{~h}$; ii. $\mathrm{PhCOCl}\left(4.8\right.$ equiv), $<40^{\circ} \mathrm{C}$, $24 \mathrm{~h}$ (71\% for two steps); (b) 1:1:0.6:0.18 $\mathrm{CH}_{2} \mathrm{Cl}_{2}-\mathrm{HOAc}-$ $\mathrm{Ac}_{2} \mathrm{O}-\mathrm{H}_{2} \mathrm{SO}_{4}, \mathrm{rt}, 20 \mathrm{~h}(83 \%)$; (c) benzylamine (4.0 equiv), THF, rt, $24 \mathrm{~h}(88 \%)$; (d) $\mathrm{CCl}_{3} \mathrm{CN}$ (3.3 equiv), DBU (0.3 equiv), rt, 5 h $(86 \%)$. structure of the tetrasaccharide 11. Selective removal of the 6-O-acetyl group of the tetrasaccharide $\mathbf{1 1}$ gave the glycosyl acceptor 13. Deallylation ${ }^{9}$ of 11 with $\mathrm{PdCl}_{2}$, followed by activation with $\mathrm{CCl}_{3} \mathrm{CN}$ in the presence of $\mathrm{K}_{2} \mathrm{CO}_{3}$, gave the tetrasaccharide donor 12. The fully protected octasaccharide $\mathbf{1 4}$ was smoothly obtained by coupling 13 with $\mathbf{1 2}$. The ${ }^{13} \mathrm{C}$ NMR spectrum of $\mathbf{1 4}$ gave eight signals for C-1 (100.08, 100.06, 98.9, 98.7, 98.1, 97.8, 97.6, 96.8 (8 C-1). Finally, deacylation of $\mathbf{1 4}$ in ammonia-saturated methanol gave the target octasaccharide 1. A bioassay of $\mathbf{1}$ for its immunoinhibitory activity is in progress.

In summary, a highly efficient and concise synthesis of the mannose octasaccharide repeating unit of the mannans of the cell-walls of some fungi was achieved by regio- and stereoselective glycosylation using glycosyl trichloroacetimidates as the donors and partially protected sugars as the acceptors. The sole use of acyl groups in the synthesis substantially simplified the procedure. This method should also be useful for the synthesis of high-mannose oligosaccharides.

\section{Experimental}

General methods.-Optical rotations were determined at $25^{\circ} \mathrm{C}$ with a Perkin-Elmer model 241-Mc automatic polarimeter. Melting points were determined with a 'Mel-Temp' apparatus. ${ }^{1} \mathrm{H}$ NMR and ${ }^{13} \mathrm{C}$ NMR spectra were recorded with Bruker ARX 400 spectrometers $\left(400 \mathrm{MHz}\right.$ for ${ }^{1} \mathrm{H}, 100 \mathrm{MHz}$ for ${ }^{13} \mathrm{C}$ ) for solutions in $\mathrm{CDCl}_{3}$ or $\mathrm{D}_{2} \mathrm{O}$ as indicated. Chemical shifts are given in ppm downfield from internal $\mathrm{Me}_{4} \mathrm{Si}$. Mass spectra were recorded with a VG PLATFORM mass spectrometer in the ESI mode. Thin-layer chromatography (TLC) was performed on silica gel $\mathrm{HF}_{254}$ with detection by charring with $30 \%(\mathrm{v} / \mathrm{v}) \mathrm{H}_{2} \mathrm{SO}_{4}$ in $\mathrm{MeOH}$ or in some cases by a UV detector. Column chromatography was conducted by elution of a column $(16 \times 240$ $\mathrm{mm}, 18 \times 300 \mathrm{~mm}, 35 \times 400 \mathrm{~mm})$ of silica gel $(100-200$ mesh) with EtOAc-petroleum ether $\left(60-90^{\circ} \mathrm{C}\right)$ as the eluent. Solutions were concentrated at $<60^{\circ} \mathrm{C}$ under reduced pressure.

3,4-Di-O-benzoyl-1,2-O-[(R)-ethylidene]-6-O-trityl$\beta$ - D - mannopyranose (3). - A solution of 1,2-O-[(R)ethylidene]- $\beta$-D-mannopyranose $(2)^{7}(6.4 \mathrm{~g}, 31.1 \mathrm{mmol})$ and trityl chloride $(9.8 \mathrm{~g}, 35.0 \mathrm{mmol})$ in pyridine $(100$ $\mathrm{mL}$ ) was stirred at $50^{\circ} \mathrm{C}$ for $32 \mathrm{~h}$, at the end of which time TLC (1:1 petroleum ether-EtOAc) indicated that the reaction was complete. The reaction mixture was cooled to $0{ }^{\circ} \mathrm{C}$, and then benzoyl chloride $(7.9 \mathrm{~mL}, 68$ mmol) was added dropwise within 30 min to keep the reaction temperature under $40^{\circ} \mathrm{C}$. After $24 \mathrm{~h}$, water $(300 \mathrm{~mL})$ was added to the reaction mixture, and stirring was continued for $30 \mathrm{~min}$. The mixture was extracted with $\mathrm{CH}_{2} \mathrm{Cl}_{2}(3 \times 100 \mathrm{~mL})$, and the combined 

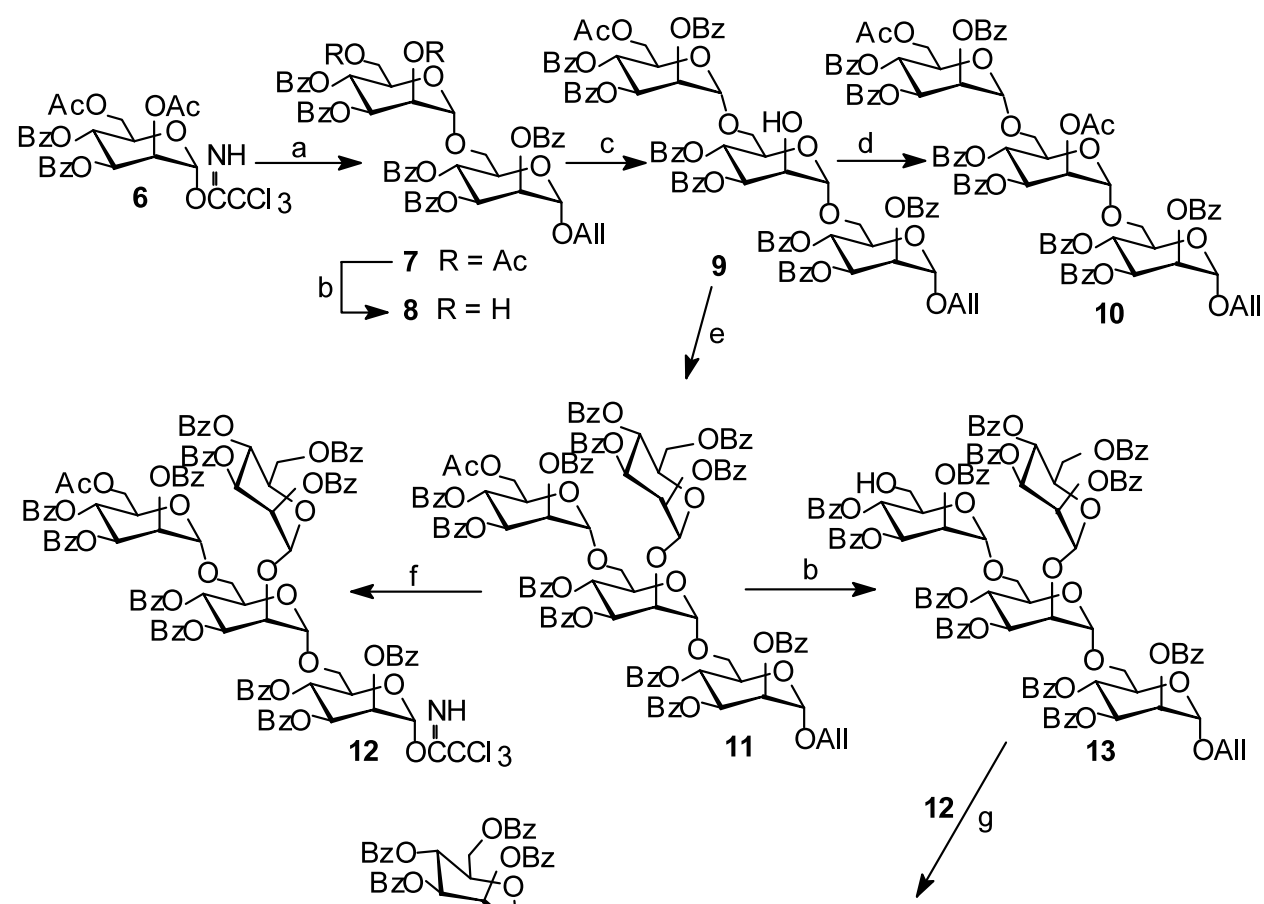

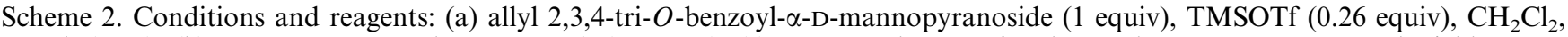
rt, $3 \mathrm{~h}$ (88\%); (b) $\mathrm{MeOH}-0.5 \% \mathrm{HCl}, \mathrm{rt}, 12-14 \mathrm{~h}(93-96 \%$ ); (c) 6- $O$-acetyl-2,3,4-tri- $O$-benzoyl- $\alpha$-D-mannopyranosyl trichloroacetimidate (1.0 equiv), $\mathrm{CH}_{2} \mathrm{Cl}_{2}$, TMSOTf (0.08 equiv), rt, $3 \mathrm{~h}(86 \%)$; (d) (Ac) $2 \mathrm{O}$, pyridine, rt, $5 \mathrm{~h}(100 \%)$; (e) 2,3,4,6-tetra- $O$-benzoyl$\alpha$-D-mannopyranosyl trichloroacetimidate (1.4 equiv), $\mathrm{CH}_{2} \mathrm{Cl}_{2}, \mathrm{TMSOTf}\left(0.16\right.$ equiv), rt, $3 \mathrm{~h}(85 \%) ;(\mathrm{f}) \mathrm{i}$. $\mathrm{PdCl}_{2}, \mathrm{CH}_{3} \mathrm{OH}-\mathrm{CH}_{2} \mathrm{Cl}_{2}$, $2 \mathrm{~h}$; ii: $\mathrm{CCl}_{3} \mathrm{CN}, \mathrm{DBU}, \mathrm{CH}_{2} \mathrm{Cl}_{2} 8 \mathrm{~h}(78 \%)$; (g) 12 (1.2 equiv), $\mathrm{CH}_{2} \mathrm{Cl}_{2}$, TMSOTf (0.3 equiv), rt, 3 h (80\%); (h) $\mathrm{CH}_{3} \mathrm{OH}$ satd with dry $\mathrm{NH}_{3}$, rt, $72 \mathrm{~h}(98 \%)$.

extracts were washed with $1 \mathrm{~N} \mathrm{HCl}$ and satd aq $\mathrm{NaHCO}_{3}$, dried $\left(\mathrm{Na}_{2} \mathrm{SO}_{4}\right)$ and concentrated to a syrup that was subjected to column chromatography with $4: 1$ petroleum ether-EtOAc as the eluent. Compound 3 $(14.5 \mathrm{~g}, 71 \%)$ was obtained as a syrup: $[\alpha]_{\mathrm{D}}-37.2^{\circ}(c$ 1.0, $\left.\mathrm{CHCl}_{3}\right) ;{ }^{1} \mathrm{H}$ NMR $\left(400 \mathrm{MHz}, \mathrm{CDCl}_{3}\right): \delta 8.01,7.90$ $(2 \mathrm{~d}, 4 \mathrm{H}, \mathrm{BzH}), 7.65-7.08(\mathrm{~m}, 21 \mathrm{H}, \mathrm{Ph} H), 5.97$ (dd, $\left.1 \mathrm{H}, J_{3,4}=J_{5,4} 10.0 \mathrm{~Hz}, \mathrm{H}-4\right), 5.48\left(\mathrm{dd}, 1 \mathrm{H}, J_{2,3} 3.6 \mathrm{~Hz}\right.$, $\left.J_{4,3} 10.0 \mathrm{~Hz}, \mathrm{H}-3\right), 5.43\left(\mathrm{~d}, 1 \mathrm{H}, J_{2,1} 2.0 \mathrm{~Hz}, \mathrm{H}-1\right), 5.37$ $\left(\mathrm{q}, J 4.8 \mathrm{~Hz}, \mathrm{CH}-\mathrm{CH}_{3}\right), 4.49\left(\mathrm{dd}, J_{1,2} 2.0 \mathrm{~Hz}, J_{3,2} 3.6 \mathrm{~Hz}\right.$, $\mathrm{H}-2), 3.79\left(\mathrm{~m}, 1 \mathrm{H}, J_{6 \mathrm{a}, 5} 2.8 \mathrm{~Hz}, J_{6 \mathrm{~b}, 5} 4.4 \mathrm{~Hz}, J_{4,5} 10.0\right.$ $\mathrm{Hz}, \mathrm{H}-5), 3.37\left(\mathrm{dd}, 1 \mathrm{H}, J_{6 \mathrm{a}, 6 \mathrm{~b}} 10.2 \mathrm{~Hz}, J_{5,6 \mathrm{a}} 2.8 \mathrm{~Hz}\right.$, H-6a), 3.22 (dd, $1 \mathrm{H}, J_{6 \mathrm{a}, 6 \mathrm{~b}} 10.2 \mathrm{~Hz}, J_{5,6 \mathrm{~b}} 4.4 \mathrm{~Hz}, \mathrm{H}-6 \mathrm{~b}$ ), $1.60\left(\mathrm{~d}, 3 \mathrm{H}, J 4.8 \mathrm{~Hz}, \mathrm{CH}-\mathrm{CH}_{3}\right)$. Anal. Calcd for $\mathrm{C}_{41} \mathrm{H}_{36} \mathrm{O}_{8}$ : C, 74.99; H, 5.52. Found: C, 75.16; H, 5.55.

1,2,6-Tri-O-acetyl-3,4-di-O-benzoyl- $\alpha$-D-mannopyranose (4). - Compound 3 (11 g, $16.77 \mathrm{~mol})$ was dissolved in a mixture of $\mathrm{CH}_{2} \mathrm{Cl}_{2}(50 \mathrm{~mL}), \mathrm{Ac}_{2} \mathrm{O}(50 \mathrm{~mL})$ and $\mathrm{AcOH}(30 \mathrm{~mL})$, the solution was cooled to $10^{\circ} \mathrm{C}$ in an ice bath, and $\mathrm{H}_{2} \mathrm{SO}_{4}(8.8 \mathrm{~mL})$ was added dropwise over $20 \mathrm{~min}$. After the addition was complete, the ice bath was removed and the reaction was allowed to continue for 20 $\mathrm{h}$ at ambient temperature. The reaction solution was poured into ice water $(400 \mathrm{~mL})$, stirring was continued for an additional $15 \mathrm{~min}$, and the mixture was extracted with $\mathrm{CHCl}_{3}(3 \times 100 \mathrm{~mL})$. The combined extracts were washed with $10 \%$ aq $\mathrm{NaHCO}_{3}(3 \times 60 \mathrm{~mL})$, dried over $\mathrm{Na}_{2} \mathrm{SO}_{4}$, and concentrated to a syrup that was subjected to column chromatography with $4: 1$ petroleum etherEtOAc as the eluent. Compound $4(7.2 \mathrm{~g}, 83 \%)$ was obtained as a syrup: $[\alpha]_{\mathrm{D}}-28.2^{\circ}\left(c 1.0, \mathrm{CHCI}_{3}\right) ;{ }^{1} \mathrm{H}$ NMR $\left(400 \mathrm{MHz}, \mathrm{CDCl}_{3}\right): \delta 7.95,7.89(2 \mathrm{~d}, 4 \mathrm{H}, \mathrm{BzH})$, $7.53-7.34(\mathrm{~m}, 6 \mathrm{H}, \mathrm{Bz} H), 6.21\left(\mathrm{~d}, 1 \mathrm{H}, J_{2,1} 1.9 \mathrm{~Hz}, \mathrm{H}-1\right)$, $5.84\left(\mathrm{dd}, 1 \mathrm{H}, J_{3,4}=J_{5,4} 9.9 \mathrm{~Hz}, \mathrm{H}-4\right), 5.75\left(\mathrm{dd}, 1 \mathrm{H}, J_{2,3}\right.$ $\left.4.7 \mathrm{~Hz}, J_{4,3} 9.9 \mathrm{~Hz}, \mathrm{H}-3\right), 5.48\left(\mathrm{dd}, 1 \mathrm{H}, J_{1,2} 1.9 \mathrm{~Hz}, J_{3,2}\right.$ $4.7 \mathrm{~Hz}, \mathrm{H}-2), 4.36-4.20$ (m, $3 \mathrm{H}, \mathrm{H}-5, \mathrm{H}-6 \mathrm{a}, \mathrm{H}-6 \mathrm{~b}), 2.24$, 2.18, $2.05\left(3 \mathrm{~s}, 9 \mathrm{H}, 3 \mathrm{COCH}_{3}\right)$. Anal. Calcd for $\mathrm{C}_{26} \mathrm{H}_{26} \mathrm{O}_{11}: \mathrm{C}, 60.70 ; \mathrm{H}, 5.09$. Found: C, 60.98; H, 5.15. 
2,6-Di-O-acetyl-3,4-di-O-benzoyl- $\alpha$-D-mannopyranose (5). - A solution of compound $4(5 \mathrm{~g}, 9.73 \mathrm{mmol})$ and benzylamine ( $3 \mathrm{~mL}, 27.4 \mathrm{mmol})$ in anhyd THF $(30 \mathrm{~mL})$ was stirred at $\mathrm{rt}$ for $24 \mathrm{~h}$, at the end of which time TLC (3:1 petroleum ether-EtOAc) indicated that the reaction was complete. The solution was then concentrated. Purification by flash column chromatography on silica gel (3:1 petroleum ether-EtOAc) gave compound $\mathbf{5}$ as a syrup $(3.96 \mathrm{~g}, 88 \%):[\alpha]_{\mathrm{D}}-37.4^{\circ}\left(c 0.6, \mathrm{CHCl}_{3}\right) ;{ }^{1} \mathrm{H}$ NMR $\left(400 \mathrm{MHz}, \mathrm{CDCl}_{3}\right): \delta 7.95,7.89(2 \mathrm{~d}, 4 \mathrm{H}, \mathrm{BzH})$, 7.52-7.32 (m, $6 \mathrm{H}, \mathrm{BzH}), 5.84-5.76$ (m, $2 \mathrm{H}, \mathrm{H}-3, \mathrm{H}-4)$, $5.50\left(\mathrm{dd}, 1 \mathrm{H}, J_{1,2} 1.8 \mathrm{~Hz}, J_{3,2} 2.9 \mathrm{~Hz}, \mathrm{H}-2\right), 5.38(\mathrm{~d}, 1$ $\left.\mathrm{H}, J_{2,1} 1.8 \mathrm{~Hz}, \mathrm{H}-1\right), 4.51-4.24$ (m, $3 \mathrm{H}, \mathrm{H}-5, \mathrm{H}-6 \mathrm{a}, \mathrm{H}-6$ ), 2.17, $2.06\left(2 \mathrm{~s}, 6 \mathrm{H}, 2 \mathrm{COCH}_{3}\right)$. Anal. Calcd for $\mathrm{C}_{24} \mathrm{H}_{24} \mathrm{O}_{10}: \mathrm{C}, 61.01 ; \mathrm{H}, 5.12$. Found: $\mathrm{C}, 61.29 ; \mathrm{H}, 5.19$.

2,6-Di-O-acetyl-3,4-di-O-benzoyl- $\alpha$-D-mannopyranosyl trichloroacetimidate (6). - A mixture of 5 (4.0 g, 8.47 $\mathrm{mmol}), \mathrm{CCl}_{3} \mathrm{CN}(2.1 \mathrm{~mL}, 20.9 \mathrm{mmol})$, and 1,8-diazabicyclo[5.4.0]undecene (DBU) $(0.25 \mathrm{~mL}, 1.67 \mathrm{mmol})$ in dry $\mathrm{CH}_{2} \mathrm{Cl}_{2}(25 \mathrm{~mL})$ was stirred under $\mathrm{N}_{2}$ for $5 \mathrm{~h}$ and then concentrated. The residue was purified by flash chromatography (4:1 petroleum ether-EtOAc) to give 6 (4.5 g, 86\%) as a syrup: $[\alpha]_{\mathrm{D}}-19.8^{\circ}\left(c 1.0, \mathrm{CHCl}_{3}\right) ;{ }^{1} \mathrm{H}$ NMR (400 MHz, $\left.\mathrm{CDCl}_{3}\right): \delta 8.84(\mathrm{~s}, 1 \mathrm{H}, \mathrm{NH}), 7.96,7.89$ $(2 \mathrm{~d}, 4 \mathrm{H}, \mathrm{Bz} H), 7.53-7.34(\mathrm{~m}, 6 \mathrm{H}, \mathrm{Bz} H), 6.41(\mathrm{~d}, 1 \mathrm{H}$, $\left.J_{2,1} 1.8 \mathrm{~Hz}, \mathrm{H}-1\right), 5.89\left(\mathrm{dd}, 1 \mathrm{H}, J_{3,4}=J_{5,4} 10.0 \mathrm{~Hz}, \mathrm{H}-4\right)$, $5.80\left(\mathrm{dd}, 1 \mathrm{H}, J_{2,3} 3.4 \mathrm{~Hz}, J_{4,3} 10.0 \mathrm{~Hz}, \mathrm{H}-3\right), 5.70(\mathrm{dd}$, $\left.1 \mathrm{H}, J_{1,2} 1.8 \mathrm{~Hz}, J_{3,2} 3.4 \mathrm{~Hz}, \mathrm{H}-2\right), 4.46-4.26(\mathrm{~m}, 3 \mathrm{H}$, $\mathrm{H}-5, \mathrm{H}-6 \mathrm{a}, \mathrm{H}-6 \mathrm{~b}), 2.18,2.04\left(2 \mathrm{~s}, 6 \mathrm{H}, 2 \mathrm{COCH}_{3}\right)$. Anal. Calcd for $\mathrm{C}_{26} \mathrm{H}_{24} \mathrm{Cl}_{3} \mathrm{NO}_{10}$ : C, 50.62; $\mathrm{H}, 3.92$. Found: $\mathrm{C}$, $50.79 ; \mathrm{H}, 3.85$.

Allyl 2,6-di-O-acetyl-3,4-di-O-benzoyl- $\alpha$-D-mannopyranosyl-(1 $\rightarrow 6)-2,3,4$-tri-O-benzoyl- $\alpha-\mathrm{D}-$ mannopyranoside (7). - A solution of 6 (2.5 g, $4.06 \mathrm{mmol})$ and allyl 2,3,4-tri- $O$-benzoyl- $\alpha$-D-mannopyranoside $(2.16 \mathrm{~g}, 4.0$ mmol) in dry $\mathrm{CH}_{2} \mathrm{Cl}_{2}(40 \mathrm{~mL})$ was stirred with dried molecular sieves ( $4 \AA, 1 \mathrm{~g}$ ) under $\mathrm{N}_{2}$ for $15 \mathrm{~min}$, and then TMSOTf $(0.2 \mathrm{~mL}, 1.1 \mathrm{mmol})$ was added dropwise. After $1 \mathrm{~h}$ the reaction mixture was diluted with $\mathrm{CH}_{2} \mathrm{Cl}_{2}(50$ $\mathrm{mL})$ and washed with satd aq $\mathrm{NaHCO}_{3}(15 \mathrm{~mL})$. The organic layer was dried over $\mathrm{Na}_{2} \mathrm{SO}_{4}$ and concentrated. Purification of the residue by flash chromatography (3:1 petroleum ether-EtOAc) gave 7 as a syrup $(3.47 \mathrm{~g}, 88 \%)$ : $[\alpha]_{\mathrm{D}}-51.4^{\circ}\left(c 1.0, \mathrm{CHCl}_{3}\right) ;{ }^{1} \mathrm{H}$ NMR $(400 \mathrm{MHz}$, $\left.\mathrm{CDCl}_{3}\right): \delta 8.14,7.99,7.97,7.89,7.84(5 \mathrm{~d}, 10 \mathrm{H}, \mathrm{Bz} H)$, 7.55-7.27 (m, $15 \mathrm{H}, \mathrm{Bz} H), 5.99\left(\mathrm{~m}, 1 \mathrm{H}, \mathrm{CH}=\mathrm{CH}_{2}\right)$, 5.97-5.73 (m, $5 \mathrm{H}, 2 \mathrm{H}-4,2 \mathrm{H}-3, \mathrm{H}-2), 5.54(\mathrm{dd}, 1 \mathrm{H}$, $\mathrm{H}-2), 5.53\left(\mathrm{dd}, 1 \mathrm{H},{ }^{2} J 1.3 \mathrm{~Hz},{ }^{3} J_{\text {trans }} 17.1 \mathrm{~Hz}, \mathrm{CH}=\mathrm{CH}_{2}\right)$, $5.35\left(\mathrm{dd}, 1 \mathrm{H},{ }^{2} J 1.3 \mathrm{~Hz},{ }^{3} J_{\text {cis }} 10.4 \mathrm{~Hz}, \mathrm{CH}=\mathrm{CH}_{2}\right), 5.16$ $\left(\mathrm{d}, 1 \mathrm{H}, J_{2^{\prime}, 1^{\prime}} 1.5 \mathrm{~Hz}, \mathrm{H}-1^{\prime}\right), 4.97\left(\mathrm{~d}, 1 \mathrm{H}, J_{2,1} 1.5 \mathrm{~Hz}, \mathrm{H}-1\right)$, 4.40-3.70 (m, $\left.8 \mathrm{H}, \mathrm{CH}_{2} \mathrm{CH}=\mathrm{CH}_{2}, 2 \mathrm{H}-5,4 \mathrm{H}-6\right), 2.13$, $1.88\left(2 \mathrm{~s}, 6 \mathrm{H}, 2 \mathrm{COCH}_{3}\right)$. Anal. Calcd for $\mathrm{C}_{54} \mathrm{H}_{50} \mathrm{O}_{18}$ : C, 65.72; H, 5.10. Found: C, 65.94; H, 5.07.

Allyl 3,4-di-O-benzoyl- $\alpha$-D-mannopyranosyl- $(1 \rightarrow 6)$ 2,3,4-tri-O-benzoyl- $\alpha$-D-mannopyranoside $(\mathbf{8})$. - A solution of $7(1.6 \mathrm{~g}, 1.62 \mathrm{mmol})$ in aq $\mathrm{MeOH}(80 \mathrm{~mL})$ containing $0.5 \% \mathrm{HCl}$ was stirred at $\mathrm{rt}$ for $12 \mathrm{~h}$, at the end of which time TLC (2:1 petroleum ether-EtOAc) indicated that the starting material had disappeared. The mixture was neutralized with $\mathrm{Et}_{3} \mathrm{~N}$ and then concentrated to dryness. The residue was partitioned between water and $\mathrm{CH}_{2} \mathrm{Cl}_{2}$, then the organic layer was dried over $\mathrm{Na}_{2} \mathrm{SO}_{4}$ and concentrated to a syrup. Purification of the residue by flash chromatography (2:1 petroleum etherEtOAc) gave 8 as a syrup $(1.36 \mathrm{~g}, 93 \%):[\alpha]_{\mathrm{D}}-8.8^{\circ}(c$ 1.0, $\mathrm{CHCl}_{3}$ ); ${ }^{1} \mathrm{H} \mathrm{NMR}\left(400 \mathrm{MHz}, \mathrm{CDCl}_{3}\right): \delta 8.15,8.05$, 8.00, 7.96, $7.89(5 \mathrm{~d}, 10 \mathrm{H}, \mathrm{BzH}), 7.53-7.25(\mathrm{~m}, 15 \mathrm{H}$, $\mathrm{BzH}), 6.06-5.71\left(\mathrm{~m}, 6 \mathrm{H}, \mathrm{CH}=\mathrm{CH}_{2}, 2 \mathrm{H}-4,2 \mathrm{H}-3,1\right.$ $\mathrm{H}-2), 5.48\left(\mathrm{dd}, 1 \mathrm{H},{ }^{2} J 1.4 \mathrm{~Hz},{ }^{3} J_{\text {trans }} 17.2 \mathrm{~Hz}, \mathrm{CH}=\mathrm{CH}_{2}\right)$, $5.38\left(\mathrm{dd}, 1 \mathrm{H},{ }^{2} \mathrm{~J} 1.4 \mathrm{~Hz},{ }^{3} J_{\text {cis }} 10.4 \mathrm{~Hz}, \mathrm{CH}=\mathrm{CH}_{2}\right), 5.15$ $\left(\mathrm{d}, 1 \mathrm{H}, J_{2,1} 1.5 \mathrm{~Hz}, \mathrm{H}-1\right), 5.05\left(\mathrm{~d}, 1 \mathrm{H}, J_{2^{\prime}, 1^{\prime}} 1.4 \mathrm{~Hz}, \mathrm{H}-1^{\prime}\right)$, 4.41-3.46 (m, $\left.9 \mathrm{H}, \mathrm{CH}_{2} \mathrm{CH}=\mathrm{CH}_{2}, 2 \mathrm{H}-5,4 \mathrm{H}-6,1 \mathrm{H}-2\right)$. Anal. Calcd for $\mathrm{C}_{50} \mathrm{H}_{46} \mathrm{O}_{16}: \mathrm{C}, 66.51 ; \mathrm{H}, 5.13$. Found: $\mathrm{C}$, 66.80; H, 5.19.

Allyl 6-O-acetyl-2,3,4-tri-O-benzoyl- $\alpha$-D-mannopyranosyl-(1 $\rightarrow 6)-3,4-d i$-O-benzoyl- $\alpha$-D-mannopyranosyl$(1 \rightarrow 6)$-2,3,4-tri-O-benzoyl- $\alpha$-D-mannopyranoside (9).A solution of $8(0.37 \mathrm{~g}, 0.41 \mathrm{mmol})$ and TMSOTf $(10 \mu \mathrm{L}$, $0.055 \mathrm{mmol})$ in dry $\mathrm{CH}_{2} \mathrm{Cl}_{2}(6 \mathrm{~mL})$ was stirred with dried molecular sieves ( $4 \AA, 0.4 \mathrm{~g}$ ) under $\mathrm{N}_{2}$ for $15 \mathrm{~min}$, and then 6- $O$-acetyl-2,3,4-tri- $O$-benzoyl- $\alpha$-D-mannopyranosyl trichloroacetimidate $(0.28 \mathrm{~g}, 0.41 \mathrm{mmol})$ in $\mathrm{CH}_{2} \mathrm{Cl}_{2}$ $(4 \mathrm{~mL})$ was added dropwise within $20 \mathrm{~min}$. After $3 \mathrm{~h}$ the reaction mixture was diluted with $\mathrm{CH}_{2} \mathrm{Cl}_{2}(30 \mathrm{~mL})$ and washed with satd aq $\mathrm{NaHCO}_{3}(5 \mathrm{~mL})$. The organic layer was dried over $\mathrm{Na}_{2} \mathrm{SO}_{4}$ and concentrated. Purification of the residue by flash chromatography (2:1 petroleum ether-EtOAc) gave 9 as a syrup $(0.50 \mathrm{~g}, 86 \%)$ : $[\alpha]_{\mathrm{D}}$ $-51.4^{\circ}\left(c 1.0, \mathrm{CHCl}_{3}\right) ;{ }^{1} \mathrm{H}$ NMR $\left(400 \mathrm{MHz}, \mathrm{CDCl}_{3}\right): \delta$ 8.08-7.25 (m, 40 H, 8 BzH), 6.26-5.77 (m, 9 H, $\left.\mathrm{CH}=\mathrm{CH}_{2}, 3 \mathrm{H}-4,3 \mathrm{H}-3,2 \mathrm{H}-2\right), 5.41\left(\mathrm{dd}, 1 \mathrm{H},{ }^{2} J 1.4 \mathrm{~Hz}\right.$, $\left.{ }^{3} J_{\text {trans }} 17.2 \mathrm{~Hz}, \mathrm{CH}=\mathrm{CH}_{2}\right), 5.25\left(\mathrm{dd}, 1 \mathrm{H},{ }^{2} J 1.4 \mathrm{~Hz},{ }^{3} J_{\text {cis }}\right.$ $\left.10.4 \mathrm{~Hz}, \mathrm{CH}=\mathrm{CH}_{2}\right), 5.20\left(\mathrm{~d}, 1 \mathrm{H}, J_{2,1} 1.4 \mathrm{~Hz}, \mathrm{H}-1\right), 5.14$ (d, $\left.1 \mathrm{H}, J_{2,1} 1.3 \mathrm{~Hz}, \mathrm{H}-1\right), 4.84$ (d, $\left.1 \mathrm{H}, J 1.4 \mathrm{~Hz}, \mathrm{H}-1\right)$, 4.47-3.37 (m, $13 \mathrm{H}, \mathrm{CH}_{2} \mathrm{CH}=\mathrm{CH}_{2}, 1 \mathrm{H}-2,3 \mathrm{H}-5,6 \mathrm{H}-6$, $\mathrm{OH}), 2.00\left(\mathrm{~s}, 3 \mathrm{H}, \mathrm{COCH}_{3}\right)$. Anal. Calcd for $\mathrm{C}_{79} \mathrm{H}_{70} \mathrm{O}_{25}$. C, 66.85; H, 4.97. Found: C, 66.98; H, 4.86.

Allyl 6-O-acetyl-2,3,4-tri-O-benzoyl- $\alpha$-D-mannopyranosyl-(1 $\rightarrow 6)$-2-O-acetyl-3,4-di-O-benzoyl- $\alpha$-D-mannopyranosyl-( $1 \rightarrow 6)$-2,3,4-tri-O-benzoyl- $\alpha$-D-mannopyranoside (10). - To a solution of $9(1.20 \mathrm{~g}, 0.84 \mathrm{mmol})$ in pyridine $(20 \mathrm{~mL}), \mathrm{Ac}_{2} \mathrm{O}(1 \mathrm{~mL}, 10 \mathrm{mmol})$ was added dropwise, and the mixture was stirred overnight at $\mathrm{rt}$. TLC (2:1 petroleum ether-EtOAc) indicated that the reaction was complete. The mixture was diluted with $\mathrm{CH}_{2} \mathrm{Cl}_{2}$ and sequentially washed with $1 \mathrm{~N} \mathrm{HCl}$, water, and satd aq $\mathrm{NaHCO}_{3}$. The organic layers were combined, dried, and concentrated. Purification by column chromatography (2:1 petroleum ether-EtOAc) quantitatively gave 10 as a syrup: $[\alpha]_{\mathrm{D}}-99.6^{\circ}\left(c 1.0, \mathrm{CHCl}_{3}\right)$; ${ }^{1} \mathrm{H}$ NMR $\left(400 \mathrm{MHz}, \mathrm{CDCl}_{3}\right): \delta 8.08-7.24(\mathrm{~m}, 40 \mathrm{H}, 8$ $\mathrm{BzH}), 6.21-5.79\left(\mathrm{~m}, 9 \mathrm{H}, \mathrm{CH}=\mathrm{CH}_{2}, 3 \mathrm{H}-4,3 \mathrm{H}-3,2\right.$ $\mathrm{H}-2), 5.67(\mathrm{dd}, 1 \mathrm{H}, \mathrm{H}-2), 5.41\left(\mathrm{dd}, 1 \mathrm{H},{ }^{2} J 1.4 \mathrm{~Hz}\right.$, 
$\left.{ }^{3} J_{\text {trans }} 17.2 \mathrm{~Hz}, \mathrm{CH}=\mathrm{CH}_{2}\right), 5.25\left(\mathrm{dd}, 1 \mathrm{H},{ }^{2} J 1.4 \mathrm{~Hz},{ }^{3} J_{\text {cis }}\right.$ $\left.10.4 \mathrm{~Hz}, \mathrm{CH}=\mathrm{CH}_{2}\right), 5.20\left(\mathrm{~d}, 1 \mathrm{H}, J_{2,1} 1.4 \mathrm{~Hz}, \mathrm{H}-1\right), 5.06$ $\left(\mathrm{d}, 1 \mathrm{H}, J_{2,1} 1.3 \mathrm{~Hz}, \mathrm{H}-1\right), 4.87\left(\mathrm{~d}, 1 \mathrm{H}, J_{2,1} 1.4 \mathrm{~Hz}\right.$, $\mathrm{H}-1), 4.42-3.42\left(\mathrm{~m}, 11 \mathrm{H}, \mathrm{CH}_{2} \mathrm{CH}=\mathrm{CH}_{2}, 3 \mathrm{H}-5,6 \mathrm{H}-6\right)$, 2.21, $2.00\left(2 \mathrm{~s}, 6 \mathrm{H}, 2 \mathrm{COCH}_{3}\right)$. Anal. Calcd for $\mathrm{C}_{81} \mathrm{H}_{72} \mathrm{O}_{26}: \mathrm{C}, 66.57 ; \mathrm{H}, 4.96$. Found: $\mathrm{C}, 66.31 ; \mathrm{H}, 5.03$. Allyl 6-O-acetyl-2,3,4-tri-O-benzoyl- $\alpha$-D-mannopyranosyl-(1 $\rightarrow 6)$-[2,3,4,6-tetra-O-benzoyl- $\alpha$-D-mannopyranosyl-(1 $\rightarrow 2)]-3,4-d i$-O-benzoyl- $\alpha$-D-mannopyranosyl$(1 \rightarrow 6)-2,3,4-t r i-O-b e n z o y l-\alpha-\mathrm{D}-m a n n o p y r a n o s i d e \quad(\mathbf{1 1})$. -A solution of $9(0.37 \mathrm{~g}, 0.26 \mathrm{mmol})$ and TMSOTf (10 $\mu \mathrm{L}, 0.055 \mathrm{mmol})$ in dry $\mathrm{CH}_{2} \mathrm{Cl}_{2}(6 \mathrm{~mL})$ was stirred with dried molecular sieves $(4 \AA, 0.4 \mathrm{~g})$ under $\mathrm{N}_{2}$ for $15 \mathrm{~min}$, and then 2,3,4,6-tetra- $O$-benzoyl- $\alpha$-D-mannopyranosyl trichloroacetimidate $(0.26 \mathrm{~g}, 0.35 \mathrm{mmol})$ in $\mathrm{CH}_{2} \mathrm{Cl}_{2}(4$ $\mathrm{mL}$ ) was added dropwise within $20 \mathrm{~min}$. After $3 \mathrm{~h}$ the reaction mixture was diluted with $\mathrm{CH}_{2} \mathrm{Cl}_{2}(30 \mathrm{~mL})$ and washed with satd aq $\mathrm{NaHCO}_{3}(5 \mathrm{~mL})$. The organic layer was dried over $\mathrm{Na}_{2} \mathrm{SO}_{4}$ and concentrated in vacuo. Purification of the residue by flash chromatography (2:1 petroleum ether-EtOAc) gave $\mathbf{1 1}$ as a syrup $(0.45 \mathrm{~g}, 85 \%):[\alpha]_{\mathrm{D}}-72.5^{\circ}\left(c 1.0, \mathrm{CHCl}_{3}\right) ;{ }^{1} \mathrm{H}$ NMR $\left(400 \mathrm{MHz}, \mathrm{CDCl}_{3}\right): \delta 8.10-7.02(\mathrm{~m}, 60 \mathrm{H}, 12 \mathrm{BzH})$, 6.20-5.67 (m, $\left.12 \mathrm{H}, \mathrm{CH}=\mathrm{CH}_{2}, 4 \mathrm{H}-4,4 \mathrm{H}-3,3 \mathrm{H}-2\right)$, $5.47\left(\mathrm{dd}, 1 \mathrm{H},{ }^{2} J 1.5 \mathrm{~Hz},{ }^{3} J_{\text {trans }} 17.1 \mathrm{~Hz}, \mathrm{CH}=\mathrm{CH}_{2}\right), 5.33$ $\left(\mathrm{d}, 1 \mathrm{H}, J_{2,1} 1.2 \mathrm{~Hz}, \mathrm{H}-1\right), 5.30\left(\mathrm{dd}, 1 \mathrm{H},{ }^{2} J 1.5 \mathrm{~Hz},{ }^{3} J_{\text {cis }}\right.$ $\left.10.4 \mathrm{~Hz}, \mathrm{CH}=\mathrm{CH}_{2}\right), 5.20(\mathrm{~d}, 1 \mathrm{H}, J 1.4 \mathrm{~Hz}, \mathrm{H}-1), 5.05$ $\left(\mathrm{d}, 1 \mathrm{H}, J_{2,1} 1.4 \mathrm{~Hz}, \mathrm{H}-1\right), 4.91\left(\mathrm{~d}, 1 \mathrm{H}, J_{2,1} 1.4 \mathrm{~Hz}\right.$, $\mathrm{H}-1)$, 4.77-3.49 (m, $15 \mathrm{H}, \mathrm{CH}_{2} \mathrm{CH}=\mathrm{CH}_{2}, \mathrm{H}-2,4 \mathrm{H}-5,8$ $\mathrm{H}-6), 1.96\left(\mathrm{~s}, 3 \mathrm{H}, \mathrm{COCH}_{3}\right) ;{ }^{13} \mathrm{C} \mathrm{NMR}(100 \mathrm{MHz}$, $\left.\mathrm{CDCl}_{3}\right): \delta 170.5\left(1 \mathrm{C}, \mathrm{COCH}_{3}\right), 166.0,165.7,165.7$, $165.6,165.5,165.5,165.4,165.3,165.1,164.9,164.8$, 164.7 (12 C, $12 \mathrm{COPh}), 133.3-127.7(73 \mathrm{C}, 12 \mathrm{Ph}$, $\left.\mathrm{CH}_{2} \mathrm{CH}=\mathrm{CH}_{2}\right), 118.2\left(1 \mathrm{C}, \mathrm{CH}_{2} \mathrm{CH}=\mathrm{CH}_{2}\right), 100.0,98.8$, 97.3, 96.8 (4 C-1), $20.5\left(1 \mathrm{C}, \mathrm{COCH}_{3}\right)$. Anal. Calcd for $\mathrm{C}_{113} \mathrm{H}_{96} \mathrm{O}_{34}: \mathrm{C}, 67.93 ; \mathrm{H}, 4.84$. Found: $\mathrm{C}, 67.78 ; \mathrm{H}$, 4.89 .

Allyl 2,3,4-tri-O-benzoyl- $\alpha$-D-mannopyranosyl- $(1 \rightarrow$ 6)-[2,3,4,6-tetra-O-benzoyl- $\alpha$-D-mannopyranosyl- $(1 \rightarrow$ 2)]-3,4-di-O-benzoyl- $\alpha$-D-mannopyranosyl- $(1 \rightarrow 6)$-2,3,4tri-O-benzoyl- $\alpha$-D-mannopyranoside (13). - A solution of $11(1.6 \mathrm{~g}, 0.80 \mathrm{mmol})$ in $\mathrm{MeOH}(80 \mathrm{~mL})$ containing $0.5 \% \mathrm{HCl}$ was stirred at $\mathrm{rt}$ for $12 \mathrm{~h}$, at the end of which time TLC (1:1 petroleum ether-EtOAc) indicated that the starting material had disappeared. The mixture was neutralized with $\mathrm{Et}_{3} \mathrm{~N}$ and then concentrated to dryness. The residue was partitioned between water and $\mathrm{CH}_{2} \mathrm{Cl}_{2}$, then the organic layer was dried over $\mathrm{Na}_{2} \mathrm{SO}_{4}$, and concentrated to a syrup. Purification of the residue by flash chromatography (1:1 petroleum ether-EtOAc) gave $13(1.50 \mathrm{~g}, 96 \%)$ : $[\alpha]_{\mathrm{D}}-79.6^{\circ}\left(c 1.0, \mathrm{CHCl}_{3}\right) ;{ }^{1} \mathrm{H}$ NMR $\left(400 \mathrm{MHz}, \mathrm{CDCl}_{3}\right): \delta 8.11-7.08(\mathrm{~m}, 60 \mathrm{H}, 12$ $\mathrm{BzH}), 6.21-5.60\left(\mathrm{~m}, 12 \mathrm{H}, \mathrm{CH}=\mathrm{CH}_{2}, 4 \mathrm{H}-4,4 \mathrm{H}-3,3\right.$ $\mathrm{H}-2), 5.47\left(\mathrm{dd}, 1 \mathrm{H},{ }^{2} J 1.4 \mathrm{~Hz},{ }^{3} J_{\text {trans }} 17.2 \mathrm{~Hz}\right.$, $\left.\mathrm{CH}=\mathrm{CH}_{2}\right), 5.32\left(\mathrm{~d}, 1 \mathrm{H}, J_{2,1} 1.2 \mathrm{~Hz}, \mathrm{H}-1\right), 5.30(\mathrm{dd}, 1 \mathrm{H}$, $\left.{ }^{2} J 1.4 \mathrm{~Hz},{ }^{3} J_{\text {cis }} 10.4 \mathrm{~Hz}, \mathrm{CH}=\mathrm{CH}_{2}\right), 5.23\left(\mathrm{~d}, 1 \mathrm{H}, J_{2,1} 1.3\right.$
$\mathrm{Hz}, \mathrm{H}-1), 5.05$ (d, $1 \mathrm{H}, J 1.4 \mathrm{~Hz}, \mathrm{H}-1), 4.90\left(\mathrm{~d}, 1 \mathrm{H}, J_{2,1}\right.$ $1.2 \mathrm{~Hz}, \mathrm{H}-1), 4.71-3.48\left(\mathrm{~m}, 15 \mathrm{H}, \mathrm{CH}_{2} \mathrm{CH}=\mathrm{CH}_{2}, \mathrm{H}-2,4\right.$ $\mathrm{H}-5,8 \mathrm{H}-6) ;{ }^{13} \mathrm{C}$ NMR $\left(100 \mathrm{MHz}, \mathrm{CDCl}_{3}\right): \delta$ 166.6, $166.0,165.7,165.6,165.5,165.5,165.4,165.4,165.2$, 164.9, 164.9, 164.7 (12 C, $12 \mathrm{COPh}), 133.4-127.8$ (73 $\left.\mathrm{C}, 12 \mathrm{Ph}, \mathrm{CH}_{2} \mathrm{CH}=\mathrm{CH}_{2}\right), 118.2\left(1 \mathrm{C}, \mathrm{CH}_{2} \mathrm{CH}=\mathrm{CH}_{2}\right)$, 99.9, 98.7, 97.3, 96.8 (4 C-1). Anal. Calcd for $\mathrm{C}_{111} \mathrm{H}_{94} \mathrm{O}_{33}: \mathrm{C}, 68.17 ; \mathrm{H}, 4.84$. Found: $\mathrm{C}, 68.44 ; \mathrm{H}$, 4.78 .

6-O-Acetyl-2,3,4-tri-O-benzoyl- $\alpha$-D-mannopyranosyl$(1 \rightarrow 6)$ - [2,3,4,6-tetra-O-benzoyl- $\alpha$-D-mannopyranosyl$(1 \rightarrow 2)]-3,4-d i$-O-benzoyl- $\alpha$-D-mannopyranosyl- $(1 \rightarrow 6)$ 2,3,4-tri-O-benzoyl- $\alpha$-D-mannopyranosyl trichloroacetimidate (12). - A mixture of compound 11 (2.24 g, 1.12 mmol) and $\mathrm{PdCl}_{2}(50 \mathrm{mg})$ in dry $\mathrm{MeOH}(50 \mathrm{~mL})$ (Caution! Extreme fire hazard.) was stirred vigorously for $4 \mathrm{~h}$ at $\mathrm{rt}$, TLC (1:1 petroleum ether-EtOAc) indicated that the reaction was complete. The reaction mixture was filtered through Celite, and the filtrate was concentrated to dryness. The resulting compound was dissolved in $\mathrm{CH}_{2} \mathrm{Cl}_{2}(20 \mathrm{~mL})$, then $\mathrm{CCl}_{3} \mathrm{CN}(0.3 \mathrm{~mL}, 3$ mmol) and DBU ( $42 \mu \mathrm{L}, 0.3 \mathrm{mmol})$ were added. The reaction mixture was stirred for $2 \mathrm{~h}$, at the end of which time TLC (1:1 petroleum ether-EtOAc) indicated that the reaction was complete. Concentration of the reaction mixture, followed by purification on a silica gel column with 1:1 petroleum ether-EtOAc as the eluent, furnished the tetrasaccharide donor $\mathbf{1 2}$ in good yield (1.84 g, 78\% two steps): $[\alpha]_{\mathrm{D}}-71.5^{\circ}\left(c 1.0, \mathrm{CHCl}_{3}\right) ;{ }^{1} \mathrm{H}$ NMR (400 MHz, $\left.\mathrm{CDCl}_{3}\right): \delta 8.99(\mathrm{~s}, 1 \mathrm{H}, \mathrm{NH}), 8.09-$ $7.03(\mathrm{~m}, 60 \mathrm{H}, 12 \mathrm{BzH}), 6.64\left(\mathrm{~d}, 1 \mathrm{H}, J_{2,1} 1.5 \mathrm{~Hz}, \mathrm{H}-1\right)$, 6.35-5.90 (m, $11 \mathrm{H}, 4 \mathrm{H}-4,4 \mathrm{H}-3,3 \mathrm{H}-2), 5.54(\mathrm{~d}, 1 \mathrm{H}$, $\left.J_{2,1} 1.2 \mathrm{~Hz}, \mathrm{H}-1\right), 5.31\left(\mathrm{~d}, 1 \mathrm{H}, J_{2,1} 1.3 \mathrm{~Hz}, \mathrm{H}-1\right), 5.12$ $\left(\mathrm{d}, 1 \mathrm{H}, J_{2,1} 1.4 \mathrm{~Hz}, \mathrm{H}-1\right), 4.88\left(\mathrm{~d}, 1 \mathrm{H}, J_{2,1} 1.5 \mathrm{~Hz}\right.$, H-1), 4.78-3.47 (m, 13 H, H-2, 4 H-5, 8 H-6), 2.04 (s, $\left.3 \mathrm{H}, \mathrm{COCH}_{3}\right) ;{ }^{13} \mathrm{C}$ NMR $\left(100 \mathrm{MHz}, \mathrm{CDCl}_{3}\right): \delta 170.4(1$ $\left.\mathrm{C}, \mathrm{COCH}_{3}\right), 165.9,165.6,165.6,165.5,165.4,165.3$, $165.3,165.2,165.0,164.9,164.8,164.7$ (12 C, 12 $\mathrm{COPh}), 159.7\left(1 \mathrm{C}, \mathrm{CCl}_{3}\right), 133.5-127.7(72 \mathrm{C}, 12 \mathrm{Ph})$, 99.9, 98.7, 97.1, 94.6 (4 C-1), $90.1\left(1 \mathrm{C}, \mathrm{C}(\mathrm{NH}) \mathrm{CCl}_{3}\right)$, $20.4\left(1 \mathrm{C}, \mathrm{COCH}_{3}\right)$. Anal. Calcd for $\mathrm{C}_{112} \mathrm{H}_{92} \mathrm{Cl}_{3} \mathrm{NO}_{34}$ : C, 63.99; H, 4.41. Found: C, 63.71; H, 4.48.

Allyl 6-O-acetyl-2,3,4-tri-O-benzoyl- $\alpha$-D-mannopyranosyl-( $1 \rightarrow 6)-[2,3,4,6$-tetra-O-benzoyl- $\alpha$-D-mannopyranosyl-(1 $\rightarrow 2)]-3,4-d i$-O-benzoyl- $\alpha$-D-mannopyranosyl$(1 \rightarrow 6)$-2,3,4-tri-O-benzoyl- $\alpha$-D-mannopyranosyl-(1 $\rightarrow 6)$ 2,3,4 - tri - O - benzoyl - $\alpha$ - D - mannopyranosyl - $(1 \rightarrow 6)$ [2,3,4,6-tetra-O-benzoyl- $\alpha$-D-mannopyranosyl- $(1 \rightarrow 2)]$ 3,4-di-O-benzoyl- $\alpha$ - D-mannopyranosyl- $(1 \rightarrow 6)-2,3,4$ tri-O-benzoyl- $\alpha$-D-mannopyranoside (14). - The tetrasaccharide donor $12(1.19 \mathrm{~g}, 0.57 \mathrm{mmol})$ and the tetrasaccharide acceptor $13(1.07 \mathrm{~g}, 0.55 \mathrm{mmol})$ were dried together under high vacuum for $2 \mathrm{~h}$, then dissolved in anhyd $\mathrm{CH}_{2} \mathrm{Cl}_{2}(30 \mathrm{~mL})$. TMSOTf $(30 \mu \mathrm{L})$ was added dropwise at $-20^{\circ} \mathrm{C}$ with $\mathrm{N}_{2}$ protection. The reaction mixture was stirred for $3 \mathrm{~h}$, during which time the 
reaction temperature gradually raised to ambient temperature. Then the mixture was neutralized with $\mathrm{Et}_{3} \mathrm{~N}$ and concentrated under reduced pressure to an oily residue. Purification by column chromatography $(1: 1$ petroleum ether-EtOAc) gave $14(1.72 \mathrm{~g}, 80 \%)$ as a syrup: $[\alpha]_{\mathrm{D}}-66.2^{\circ}\left(c\right.$ 1.0, $\left.\mathrm{CHCl}_{3}\right) ;{ }^{1} \mathrm{H}$ NMR $(400$ $\left.\mathrm{MHz}, \mathrm{CDCl}_{3}\right): \delta 8.11-6.88(\mathrm{~m}, 120 \mathrm{H}, 24 \mathrm{BzH}), 6.24-$ $5.76\left(\mathrm{~m}, 23 \mathrm{H}, \mathrm{CH}=\mathrm{CH}_{2}, 8 \mathrm{H}-4,8 \mathrm{H}-3,6 \mathrm{H}-2\right), 5.46$ (dd, $\left.1 \mathrm{H},{ }^{2} J 1.5 \mathrm{~Hz},{ }^{3} J_{\text {trans }} 17.1 \mathrm{~Hz}, \mathrm{CH}=\mathrm{CH}_{2}\right), 5.41(\mathrm{~d}, 1 \mathrm{H}$, $\left.J_{2,1} 1.2 \mathrm{~Hz}, \mathrm{H}-1\right), 5.28\left(\mathrm{dd}, 1 \mathrm{H},{ }^{2} J 1.5 \mathrm{~Hz},{ }^{3} J_{\text {cis }} 10.4 \mathrm{~Hz}\right.$, $\left.\mathrm{CH}=\mathrm{CH}_{2}\right), 5.25\left(\mathrm{~d}, 1 \mathrm{H}, J_{2,1} 1.3 \mathrm{~Hz}, \mathrm{H}-1\right), 5.17(\mathrm{~d}, 1 \mathrm{H}$, $\left.J_{2,1} 1.3 \mathrm{~Hz}, \mathrm{H}-1\right), 5.09\left(\mathrm{~d}, 1 \mathrm{H}, J_{2,1} 1.2 \mathrm{~Hz}, \mathrm{H}-1\right), 5.02$ (d, $\left.1 \mathrm{H}, J_{2,1} 1.2 \mathrm{~Hz}, \mathrm{H}-1\right), 5.00$ (m, $\left.2 \mathrm{H}, 2 \mathrm{H}-1\right), 4.95$ (d, $\left.1 \mathrm{H}, \quad J_{2,1} 1.2 \mathrm{~Hz}, \mathrm{H}-1\right), 4.79-3.38(\mathrm{~m}, 28 \mathrm{H}$, $\left.\mathrm{CH}_{2} \mathrm{CH}=\mathrm{CH}_{2}, 2 \mathrm{H}-2,8 \mathrm{H}-5,16 \mathrm{H}-6\right), 2.03(\mathrm{~s}, 3 \mathrm{H}$, $\left.\mathrm{COCH}_{3}\right) ;{ }^{13} \mathrm{C}$ NMR $\left(100 \mathrm{MHz}, \mathrm{CDCl}_{3}\right): \delta 170.3(1 \mathrm{C}$, $\left.\mathrm{COCH}_{3}\right), 166.0-164.6(24 \mathrm{C}, 24 \mathrm{COPh}), 133.3-127.3$ $\left(145 \mathrm{C}, 24 \mathrm{Ph}, \mathrm{CH}_{2} \mathrm{CH}=\mathrm{CH}_{2}\right), 118.1\left(1 \mathrm{C}, \mathrm{CH}_{2} \mathrm{CH}=\right.$ $\left.\mathrm{CH}_{2}\right), 100.08,100.06,98.9,98.7,98.1,97.8,97.6,96.8(8$ C-1), $20.3\left(1 \mathrm{C}, \mathrm{COCH}_{3}\right)$. Anal. Calcd for $\mathrm{C}_{221} \mathrm{H}_{184} \mathrm{O}_{66}$ : C, 68.13; H, 4.76. Found: C, 68.41; H, 4.70.

Allyl $\alpha$-D-mannopyranosyl-( $1 \rightarrow 6)-[\alpha-\mathrm{D}-$ mannopyranosyl- $(1 \rightarrow 2)]-\alpha-\mathrm{D}$-mannopyranosyl- $(1 \rightarrow 6)-\alpha$-D-mannopyranosyl- $(1 \rightarrow 6)-\alpha$ - D - mannopyranosyl - $(1 \rightarrow 6)-[\alpha-\mathrm{D}-$ mannopyranosyl- $(1 \rightarrow 2)]-\alpha$-D-mannopyranosyl- $(1 \rightarrow 6)$ $\alpha$-D-mannopyranoside (1). - A satd solution of ammonia in $\mathrm{MeOH}(40 \mathrm{~mL})$ was added to a solution of $\mathbf{1 4}$ (811 mg, $0.21 \mathrm{mmol})$ in $\mathrm{CH}_{2} \mathrm{Cl}_{2}(4 \mathrm{~mL})$. After a week at $\mathrm{rt}$, the reaction mixture was concentrated, and the residue was purified by chromatography on a Sephadex LH-20 (column $2.0 \times 30 \mathrm{~cm}$, flow $5 \mathrm{~mL} / \mathrm{min}$, about 300 $\mathrm{mL} \mathrm{MeOH}$ ) to afford the single octasaccharidic product 1 as a white amorphous powder ( $274 \mathrm{mg}, 98 \%$ ): $[\alpha]_{\mathrm{D}}+47.1^{\circ}\left(c 1.0, \mathrm{H}_{2} \mathrm{O}\right) ;{ }^{1} \mathrm{H}$ NMR $\left(\mathrm{D}_{2} \mathrm{O}, 400 \mathrm{MHz}\right)$ : $\delta 5.87\left(\mathrm{~m}, 1 \mathrm{H}, \mathrm{CH}=\mathrm{CH}_{2}\right), 5.24-5.14\left(2 \mathrm{H}, \mathrm{CH}=\mathrm{CH}_{2}\right)$, 5.00-4.79 (8 H-1); ${ }^{13} \mathrm{C}$ NMR (100 MHz, $\left.\mathrm{D}_{2} \mathrm{O}\right): 133.3$ (1 $\left.\mathrm{C}, \mathrm{CH}_{2} \mathrm{CH}=\mathrm{CH}_{2}\right), 118.2\left(1 \mathrm{C}, \mathrm{CH}_{2} \mathrm{CH}=\mathrm{CH}_{2}\right), 100.2$, 100.1, 99.2, 99.17, 99.10, 99.0, 97.8, 96.8 (8 C-1). ESIMS: Calcd for $\mathrm{C}_{51} \mathrm{H}_{86} \mathrm{O}_{41}, 1355.22$ [M]. Found, 1378.1 $(\mathrm{M}+\mathrm{Na})^{+}$.

\section{Acknowledgements}

This work was supported by the Beijing Natural Science Foundation (6021004) and National Natural Science Foundation of China (59973026 and 29905004).

\section{References}

1. Hay R. J. J. Dermatol. 1982, 106, 1-12.

2. Dahl M. V. J. Am. Acad. Dermatol. 1994, 31, S34.

3. Ikuta K.; Shibata N.; Blake J. S.; Dahl M. V.; Nelson R. D.; Hisamichi K.; Kobayashi H.; Suzuki S.; Okawa Y. Biochem. J. 1997, 323, 297-305.

4. Blake J. S.; Dahl M. V.; Herron M. J.; Nelson R. D. J. Invest. Dermatol. 1991, 96, 657-663.

5. Grando S. A.; Hostager B. S.; Dahl M. V.; Nelson R. D. J. Invest. Dermatol. 1992, 98, 876-883.

6. Grando S. A.; Hostager B. S.; Dahl M. V.; Nelson R. D. J. Invest. Dermatol. 1992, 72, 273-281.

7. Betaneli V. I.; Backinowsky L. V.; Kochetkov N. K. A. Carbohydr. Res. 1982, 107, 285-291.

8. Heng L.; Ning J.; Kong F. J. Carbohyr. Chem. 2001, 20, 285-294.

9. Boss R.; Scheffold R. Angew. Chem. 1976, 88, 578-581. 